\title{
The pressure in solute-driven surface flows
}

\author{
Diffusio-osmosis and wetting on solid surfaces: a unified description based \\ on a virtual work principle
}

Authors: Nigel Clarke, Nigel Gibbions and Didier R. Long

Soft Matter 16, 3485 (2020)

\begin{abstract}
Recommended with a Commentary by Sriram Ramaswamy, Centre for Condensed Matter Theory, Department of Physics, Indian Institute of Science, Bengaluru 560012 India
\end{abstract}

A gradient in solute concentration imposed along a uniform solid surface drives fluid flow parallel to the surface. The paper [1] highlighted in this Commentary re-examines this effect - diffusio-osmotic flow - at the heart of colloid and interfacial hydrodynamics. Classical theoretical accounts (for a review see [2]) of the phenomenon work in terms of a pressure field whose gradients in the tangential direction drive a surface slip velocity. This "pressure", of order the solute concentration times a typical interaction energy, which could mean tens to hundreds of atmospheres, does not vanish even for uniform concentration, and approaches ambient values at distances from the surface large compared to the range of the solute-surface interaction. Clarke et al. [1] point out that this is rather odd. At thermal equilibrium without gravity the pressure should be uniform and, for a single free surface, should simply be the atmospheric pressure.

Thus motivated, they take a closer look at the general theory of incompressible Stokes flow due to composition gradients. Defining a concentration field $\psi$ governed by a Gibbs freeenergy functional $G[\psi] \equiv \int_{\mathbf{r}} g(\psi, \nabla \psi \ldots)$ and chemical potential $\mu=\delta G / \delta \psi$, they calculate the change in $G$ due to a virtual displacement with compact support to argue that the operative force density due to $\psi$ is $\mu \nabla \psi$. This differs by a pure gradient from $-\psi \nabla \mu$, the form used in [2] and indeed the form that normally emerges from Poisson-brackets in the hydrodynamics of a fluid with a composition field. I return to this point at the end.

Clarke et al. [1] then extend the treatment to a fluid in contact with a solid surface, with $G \rightarrow G+\int_{\mathbf{r}} \Gamma(\psi, \mathbf{r})$, where $\Gamma(\psi, \mathbf{r})$ depends on $\psi$ near the surface, and encodes the contribution from solute-surface interactions. The virtual work argument now yields a Stokes equation with compositional force density $\left(\mu+\partial_{\psi} \Gamma\right) \nabla \psi$, and $\partial_{\psi} \Gamma \nabla \psi$ drives surface flows. They show explicitly that for a simple geometry such as a capillary open at both ends the pressure is uniform and equal to the atmospheric pressure. The mysterious confined highpressure layer in earlier treatments was clearly a result of "misfiling" a piece of the stress tensor, leading to the incorrect identification of a surface Gibbs free energy as a pressure. Calculations of the flow resulting from an imposed $\psi$ profile yield the velocity profile and the diffusio-osmotic mobility in terms of $\Gamma$, which encodes the range of the solute-surface 
interaction and the $\psi$ dependence of the surface tension. More important, in the limit of uniform concentration the equations are solved by zero velocity and uniform pressure.

The authors discuss the natural extension of their framework to describe the dynamics of wetting, the role of a slip length, and treatments in which the force density is the conventional $-\psi \nabla \mu$. Formally, one can indeed obtain the same velocity field as with $\mu \nabla \psi$, but the object that one terms the pressure in this case has a nontrivial profile and cannot be interpreted as the true pressure.

In closing, note that the force density due to $\psi$, in standard Poisson-bracket hydrodynamics, is $-\psi \nabla \mu=-\psi \nabla \delta G / \delta \psi$. This can of course be rewritten as $\mu \nabla \psi$ plus a contribution to the pressure gradient. A better prescription, however, is to write it as the divergence of a stress tensor $\boldsymbol{\sigma}$, which is always possible for a translation-invariant free-energy functional $G$, and decompose $\boldsymbol{\sigma}$ into isotropic and deviatoric (symmetric traceless) parts, $\boldsymbol{\sigma}=\boldsymbol{\sigma}_{d e v}-p \mathbf{I}$. $\boldsymbol{\sigma}_{d e v}$ is the same for the force densities $-\psi \nabla \mu$ and $\mu \nabla \psi$, and force balance $\nabla \cdot \boldsymbol{\sigma}=0$ tells us that the pressure obeys $\nabla^{2} p=\nabla \nabla: \boldsymbol{\sigma}_{\text {dev }}$ and is therefore independent of prescription. A resolution along these lines for the case of flow over a surface would I think be useful.

I thank Ananyo Maitra and Ramin Golestanian for useful discussions.

\section{References}

[1] N Clarke, N Gibbions and D R Long, Diffusio-osmosis and wetting on solid surfaces: a unified description based on a virtual work principle, Soft Matter 16, 3485 (2020).

[2] J L Anderson, Colloid transport by interfacial forces, Annu. Rev. Fluid Mech. 21, 61-99 (1989). 